\title{
Measurement of Pore Sized Microporous-Mesoporous Materials by Time-Domain Nuclear Magnetic Resonance
}

\author{
Zhi-hong Zhao, Ming-hui Zhang,* Wen-Jing Liu,* and Quan-teng Li
}

Time-domain nuclear magnetic resonance (TD NMR) technology has been used for pore detection in porous materials for a long time, but there are few pore detection methods for microporous-mesoporous materials. The surface of different materials is obtained by pore detection of known pore materials. Relaxation rate, which obtains aperture information, has an important practical significance for the application of time-domain NMR technology in the characterization of porous materials. In this study, the T2 peaks of pores of known pore size materials, namely zeolite molecular sieves $(0.3 \mathrm{~nm}$ and $1 \mathrm{~nm})$ and anodized aluminum porous membranes (30 $\mathrm{nm}$ and $90 \mathrm{~nm}$ ), were used to calculate the pore surface relaxation of zeolite molecular sieve with $0.3 \mathrm{~nm}$ pore size and $1 \mathrm{~nm}$ pore size. The ratio of the rate of the surface is 3.379; the ratio of the pore surface relaxation ratio of the $30 \mathrm{~nm}$ and $90 \mathrm{~nm}$ apertures of the anodized aluminum porous film is 3.031. This result is very close to the pore size ratio, indicating that the surface relaxation rate of the same material is directly related to the pore size, while the T2 peak can qualitatively measure the pore size.

Keywords: Nuclear magnetic resonance; Porous materials; microporous; CPMG (Carr-Purcell-MeiboomGill); Surface relaxation rate

Contact information: School of Materials Science and Art Design, Inner Mongolia Agricultural University, 306 Zhaowuda Road, Saihan District, Hohhot, Inner Mongolia, China;

*Corresponding author: 1197428998@qq.com

\section{INTRODUCTION}

Porous materials are advantageous in terms of physical and chemical properties such as high specific surface area, high porosity, strong adsorption, and easy assembly. Their uses include medical, chemical, energy storage, environmental protection, functional materials, and other fields along with being an important research object of modern material science (Ajayan et al. 2006; Levitz 2007; Song 2007; Stein et al. 2008). According to porous material aperture size (diameter, $d$ ), the porous materials are divided into three categories by IUPAC, namely, microporous $(d<2 \mathrm{~nm})$, mesoporous $(2 \mathrm{~nm}<d<50 \mathrm{~nm})$, and macroporous $(d>50 \mathrm{~nm})$ (Everett 1972). However, the study of porous materials is inseparable from the characterization and analysis of pores. The main parameters of porous materials include pore size, distribution, morphology, and channel characteristics (Kubik 1992). There are many pore characterization methods, such as mercury intrusion porosimetry (MIP), scanning electron microscopy (SEM), gas adsorption, solute exclusion, and small-angle X-ray scattering. The emerging characterization methods in recent years are atomic force microscopy (AFM), DSC thermoporosimetry (DSCT), and NMR cryoporometry (NMRC) (Yamamoto et al. 2005; Zhu et al. 2006).

Mercury intrusion porosimetry (MIP) is applied to detect the pores of porous materials, and its operation is simple. However, the pores measured by it are generally macropores, and for natural polymer porous materials such as wood, it is easy to destroy 
some thin-wall cell pores in the process of mercury intrusion, resulting in inaccurate detection results (Roels et al. 2001; Moura et al. 2005; Plötze and Niemz 2011).

Scanning electron microscopy can be used comprehensively to detect the porosity, pore size, morphology, and other information of porous materials. However, for the naturally formed polymer in porous substances, the preparation stage of the test sample may also destroy the original pore structure, and the sampling process may have rendered the detection information incomplete due to natural polymers pore inhomogeneity (Bolton et al. 1988; Wallström and Lindberg 1999).

Gas adsorption is the most commonly used characterization method for pore detection at this stage. It detects the pore size and distribution, pore morphology, and specific surface area of the material. This method usually uses nitrogen adsorption. For smaller pores, argon or krypton is also used. The slow detection speed of the sample usually takes dozens of hours and the material needs to be dry, while moisture is an important component or medium of many natural polymer materials. The gas adsorption method usually characterizes only the open pores. Wood-based porous materials and biomass porous media produce closed pores during the process of absolute drying, which can lead to a decrease in the accuracy of the characterization of porous materials by gas adsorption. Hence, it is not easily applied to biological porous materials because water is an essential part of the porous structure (Westermarck 2000; Topgaard and Söderman 2002; Papadopoulos et al. 2003; Jia et al. 2007).

The solute exclusion method involves water-saturated porous materials immersed in a solution of probe molecules that can reach equilibrium with the solution. Probe molecules enter the macropores of the porous materials and if they are small enough, they can penetrate the mesoporous. When probes enter the pores of probe molecules, they replace the water in them, which then dilutes the bulk probe solution remaining outside of the materials. The decrease in concentration of the probe solution is a measure of the pore volume of the porous materials (Telkki et al. 2013). However, the probe objects penetrate the porous materials in the manner of diffusion and cannot enter the micropores, which are smaller than the probe objects. Using probe molecules of different sizes, it is possible to obtain data for the pore size distribution of the materials (Hill 2006; Walker 2006). Most of these studies report a maximum size for swollen cell wall micropores in the region of approximately 2 to $4 \mathrm{~nm}$ (Hill 2006; Walker 2006).

Small-angle X-ray scattering (SAXS) is commonly used to characterize microporous materials, which is the degree of regularity of pore arrangement of mesoporous materials. The detection range for pores is 2 to $200 \mathrm{~nm}$. However, because this method is greatly affected by the structure of the sample, the characterization of pore size and distribution, pore structure, and specific surface area is not completely accurate (Gindl et al. 2003; Furuno et al. 2004; Gierling et al. 2005; Jeremic and Cooper 2009; Xu et al. 2013).

Atomic force microscopy (AFM) is a new pore characterization method, which can reflect microscopic information such as the pore size and morphology of the sample. It provides the three-dimensional morphology of the sample and does not require special treatment. It is recommended for testing in a variety of environments. However, atomic force microscopy has higher requirements for the flatness of the sample and the needle tip of the instrument. The imaging range is small, which still has limitations in the detection of porous materials with uneven pores (Notley and Wagberg 2005; Wang and Liu 2006).

DSC thermoporosimetry (DSCT) uses water as the medium and is based on the principle that the lower pressure at the inner surface of the pore bending in the porous 
material leads to the lower melting temperature of the ice crystal in the capillary. The relationship between the temperature reduction and the pore diameter is calculated by the Gibbs-Thomson equation. This method can obtain the porosity, pore size and distribution, pore morphology, pore volume, and other information of the material as well as achieve the measurement of the closed pore (Jiang et al. 2012a, 2012b, 2013). The sample preparation method is simple, and the degree of pore damage is small (Cavallaro et al. 2013). However, this method has higher requirements for temperature control equipment and measurement accuracy when studying water status in wood pores by DSC, which is expressed by the percentage of water content (Nakamura et al. 1981; Park et al. 2006). The pore characterization methods mentioned above have high requirements in sample preparation, detection conditions, etc., and there are still many deficiencies in the detection of natural polymers in porous materials.

Nuclear magnetic resonance is a non-destructive and fast pore characterization method in the study of porous media. In other words, the determination of pore structure by NMR is widely used in oil field exploration, finding underground water resources, crude oil structure analysis, three-dimensional structure analysis, and measurement of protein in food. Great progress has been made especially in the study of solid porous media such as soil and rock. NMR reflects pore information by measuring the NMR signal of a fluid in a porous medium. NMR signals mainly include relaxation time and free induction decay signals (Pauli et al. 2005). The signal attenuation rate is different when $\mathrm{T} 2$ is measured by NMR, because the fluid in porous material is affected by external binding conditions such as pore, pore surface, capillary, etc. A smaller pore diameter results in faster signal attenuation. The use of NMR T2 signal to study the pore structure of porous materials has begun to be applied in many fields such as geology, materials, food, and biomaterials. Li Haibo et al. (2008) compared and analyzed the mercury intrusion data and T2 distribution of the core. The distribution of the core pore radius obtained by the T2 distribution spectrum correlated with the pore throat radius distribution obtained by the mercury intrusion method. Halperin (1994) used a time-domain NMR to observe the microstructure evolution of mud hydration. The total surface area and pore size distribution during mud hydration was obtained through the T2 distribution. Wang and Wang (2013) determined the pore size distribution of the gel filtration medium by the time-domain NMR, which confirmed the feasibility of the method. Teng et al. (2014) studied the development of the water state in lignite by low temperature drying and an obtained time domain NMR to continuously monitor the change of water and pore size distribution in lignite without damage. Zhao et al. (2006) studied the conversion method and corresponding relationship between the nuclear magnetic resonance T2 distribution of tight sandstone reservoirs and the pore-throat distribution obtained by the capillary pressure curve from mercury intrusion. Zhao obtained a close correlation between them. It is effective to use the T2 distribution to evaluate the pore throat radius distribution of tight sandstone reservoirs (Zhao et al. 2006).

Magnetic resonance imaging (MRI) is representative of modern three-dimensional characterization technology. Its advantage lies in its ability to quantify the internal space of the measured substance, but its technical bottleneck has two aspects. First, the resolution of MRI imaging cannot achieve the standard that can be effectively utilized by image analysis software at this stage. This disadvantage affects the application of MRI in the characterization of porous materials. Second, the water content of the substance to be tested has certain requirements, and there are limitations in dynamically monitoring the change of material moisture. These constraints affect the application of MRI in the characterization 
of porous pores (Akoka et al. 1999; Bucur 2003; Telkki 2012).

Nuclear magnetic resonance cryoporometry (NMRC) establishes the relationship between the pore size and the freezing point of the water based on the Gibbs-Thomson equation. In other words, it reflects the pore information of the substance by measuring the different freezing points of the water in different pores. The NMRC method measures the signal returned by the freezed substance in the pore, while the DSC method measures the transient heat flow in phase transitions. The nuclear magnetic resonance technique is very sensitive in distinguishing solids from the liquids, so the measurement result has a high precision (Wang et al. 2012, 2013). This method has been used to study the saturation point and pore size distribution of wood fibers in nanoscale porous materials including pulp, membrane materials, and wood after thermal modification (Mitchell et al. 2008). It is currently unknown whether the expansion coefficient after water freezes in the pore in NMRC affects the testing results (Jackson and Makenna 1990).

In this study, nuclear magnetic resonance was used to study the pores of porous materials. The goal was to achieve a non-destructive and rapid method to determine the pore information of porous materials. A standard sample simulation method was used to determine the surface relaxivity of micropores, mesopores, and other pores. The calculation was used to simulate the pore information. The detection method is convenient and rapid because it does not require special treatment of the sample and can accurately describe pores such as micropores.

\section{EXPERIMENTAL}

\section{Materials and Equipment Materials}

There are two kinds of hydrophilic substances to be tested for measuring the surface relaxation rate. The first one is zeolite molecular sieve. The zeolite molecular sieve has a single pore size and is hydrophilic (the zeolite molecular sieve with two pore sizes is selected). The pore diameters are $0.3 \mathrm{~nm}$ and $1 \mathrm{~nm}$, respectively, labeled $3 \mathrm{~A}$ and $13 \mathrm{X}$, The selected zeolite molecular sieve is produced by China Henan Zhongbang Environmental Protection Technology Co., Ltd.; the second measured substance is anodized aluminum porous membrane (AAO). The aluminum porous membrane has a single pore size and the surface is lyophilic (selecting an anodized aluminum porous membrane of two pore sizes, pore diameters of $30 \mathrm{~nm}$ and $90 \mathrm{~nm}$, respectively, depth of $60 \mu \mathrm{m}$, labeled AAO30 and AAO90, respectively).

\section{Equipment}

T2 measurements for each sample were performed using a Bruker Minispec MQ20NMR spectrometer (Bruker, Karlsruhe, Germany). The console operates at 19.95 $\mathrm{MHz}$ inside the magnet with a probe dead-time of $4.5 \mu \mathrm{s}$. T2 measurements were performed using a CPMG (Carr-Purcell-Meiboom-Gill) sequence with 16 scans. The echo time of 0.2 ms was always used, and 300 echoes for the initial measurement were set. Depending on the specific data, data was analyzed using an Origin Pro 9.1 (version 91E, Origin Lab Corporation, Northampton, MA, USA). T2 decay curves were fitted using a third/second/first order exponential decay function. 


\section{Theory and Methods}

Theory

According to the analysis of the relaxation mechanism of T2 NMR spectroscopy, the internal water relaxation mechanism of porous materials is mainly composed of three parts: a bulk relaxation rate, a surface relaxation rate, and a diffusion relaxation rate (Xiao 1998; Toumelin et al. 2007). Equation 1 is the formula for calculating the surface relaxation rate,

$$
1 \div T 2=\left(1 \div T 2_{B}\right)+\left(1 \div T 2_{s}\right)+\left(D\left(\gamma G T_{E}\right)^{2} \div 12\right)
$$

where $T 2$ is the total relaxation time; $T 2_{B}$ is the relaxation time for water in the pores that is not in contact with the inner wall surface of the pores; and $T 2_{s}$ is the relaxation time of water in the pores in contact with the inner surface of the pores. $D\left(\gamma G T_{E}\right)^{2}$ is the relaxivity of water at the pore surface.

Under normal conditions, the solid-liquid interface interaction near the pore surface can significantly accelerate the relaxation process of water in the pore, while the relaxation efficacy of bulk water in the pore is much lower so the pore surface water provides the main $T 2$ relaxation contribution. $T 2_{B}$ is negligible (Deng 2010; Li et al. 2015). In addition, $D$ is the water diffusivity in air strikes. Usually, the diffusivity of freely moving water at room temperature is approximately 210 to $3 \mathrm{~cm}^{2} / \mathrm{s}$ (Zhao 2010), and the water diffusivity in the pores is much smaller. The parameter $\gamma$ is the proton gyromagnetic ratio 267.52 $\mathrm{MHz} / \mathrm{T}, G$ is the external magnetic field gradient, and $T_{E}$ is the callback time. When the external magnetic field is relatively uniform $(\mathrm{G} \approx 0 \mathrm{G} \mathrm{s} / \mathrm{cm}), D\left(\gamma G T_{E}\right)^{2}$ is negligible. Eq. 1 can therefore be simplified to Eq. 2.

$$
1 \div T 2 \approx 1 \div T 2_{s}
$$

Equation 3 describes surface relaxation,

$$
1 \div T 2_{s}=\rho(S \div V)
$$

where $\rho$ is the pore surface relaxivity, $S$ is the pore surface area, and $V$ is the pore volume.

Equation 4 describes $T 2$ of the moisture in the pore,

$$
1 \div T 2=\rho(S \div V)=\rho\left(F_{s} \div r\right)
$$

where $r$ is the pore radius and $F S$ is the form factor, which is generally for spherical pores $\left(F_{S}=3\right.$ ). For cylindrical pores, $F_{S}=2$ (Mao et al. 2005). Both the zeolite and AAO porous membranes in this study are cylindrical pores, resulting in Eq. 5,

$$
T 2=d \div 4 \rho
$$

where $d$ is the pore diameter.

Generally, the surface relaxation occurs on the solid-liquid contact surface and under the ideal fast diffusion limit conditions, i.e., the pores are very small and the surface relaxation is very slow, so that the molecules can travel to and from the pores several times during relaxation (Bin 2014). According to the formula, the surface relaxation rate of the sample can be calculated. 
Provencher (1982) developed a linear integral equation inversion software (CONTIN). In this paper, CONTIN software was used with the Bruker time domain NMR analyzer to invert the $T 2$ data. Among them, peaks represent states of water, and the sum of the signal amplitudes covered by the peaks represent the relative content of water in this state. The abscissa of the highest point of the peak corresponds to the mean relaxation time of water in this state.

\section{Methods}

The molecular sieve was put into a beaker to absorb water for $24 \mathrm{~h}$ to confirm its water saturation, and the molecular sieve was taken out of the water. The molecular sieve was wiped dry of surface moisture, put it into the test tube, and the time domain nuclear magnetic resonance spectrometer was used to determine $T 2$. The scan times of the time CPMG pulse sequence used for the $T 2$ measurement was 8 , cycle delay time was $2 \mathrm{~s}, \tau$ (half echo time) was $0.2 \mathrm{~ms}$, and the echo number was 300 .

AAO porous membrane was immersed into distilled water, to allow the surface to bubble for $1 \mathrm{~h}$. The surface moisture was removed, and the membrane was placed at the bottom of test tube. $T 2$ was collected and the parameter setting in the test was the same as the test parameter of the molecular sieve sample.

The natural mica sheet was placed in a beaker to absorb water for $24 \mathrm{~h}$ to determine its water absorption saturation. The mica sheet was taken out from the water to wipe dry the surface moisture, put it into a test tube, and $T 2$ was determined with a time-domain nuclear magnetic resonance spectrometer. The parameter setting in the test was the same as the test parameters of the molecular sieve and the AAO porous membrane sample. After the detection, the T2 distribution of moisture was obtained by the inversion of the T2 data collected using the CONTIN algorithm, and then the pore size analysis can be performed.

The zeolite samples $(0.3 \mathrm{~nm}$ versus $1 \mathrm{~nm})$, AAO porous membranes ( $30 \mathrm{~nm}$ versus $90 \mathrm{~nm}$ ), and natural mica sheets in the previous step were subjected to an absolute drying treatment, respectively. The pore size of the dried sample was measured by nitrogen adsorption and the BET analysis result was obtained. The $T 2$ results measured by the timedomain NMR were compared with those by nitrogen adsorption.

\section{RESULTS AND DISCUSSION}

Figure 1 shows the time-domain NMR T2 spectra measured under water-absorbing saturated conditions for $3 \mathrm{~A}$ (pore size of $0.3 \mathrm{~nm}$ ) molecular sieve sample, $13 \mathrm{X}$ (pore size of $1 \mathrm{~nm}$ ) molecular sieve sample, AAO (30) (pore size of $30 \mathrm{~nm}$ ) anodic aluminum oxide porous membrane sample, and AAO (90) (pore size of $90 \mathrm{~nm}$ ) anodic aluminum oxide porous membrane sample. The inversion data of the $T 2$ spectrum was obtained through the inversion of CONTIN software. A double exponential fit was performed on the data after inversion by Origin software to obtain the T2 spectrum. Since the sample is a single pore material, there is only one peak in this $\mathrm{T} 2$ spectrum

The $T 2$ spectrum of the $3 \mathrm{~A}$ sample in Fig. 1a had a peak apex at a position of approximately $0.3 \mathrm{~ms}$. The $T 2$ spectrum of the $13 \mathrm{X}$ sample in Fig. $1 \mathrm{~b}$ had a peak apex at a position of approximately $1 \mathrm{~ms}$. The $T 2$ spectrum of the AAO (30) sample had a peak apex at a position of approximately $3 \mathrm{~ms}$. The $T 2$ spectrum of the AAO (90) sample has a peak apex at a position of approximately $9 \mathrm{~ms}$. Table 1 shows the data presentation of the peak apex in the $8 T 2$ spectra of the four test samples. The reliability of the experiment was 
increased by averaging the values of all 8 measurements. In Table 2 the surface relaxivity was obtained by the average calculation of the $T 2$ peak apex of the sample in Table 2, which was calculated using Eqs. 1 through 5.
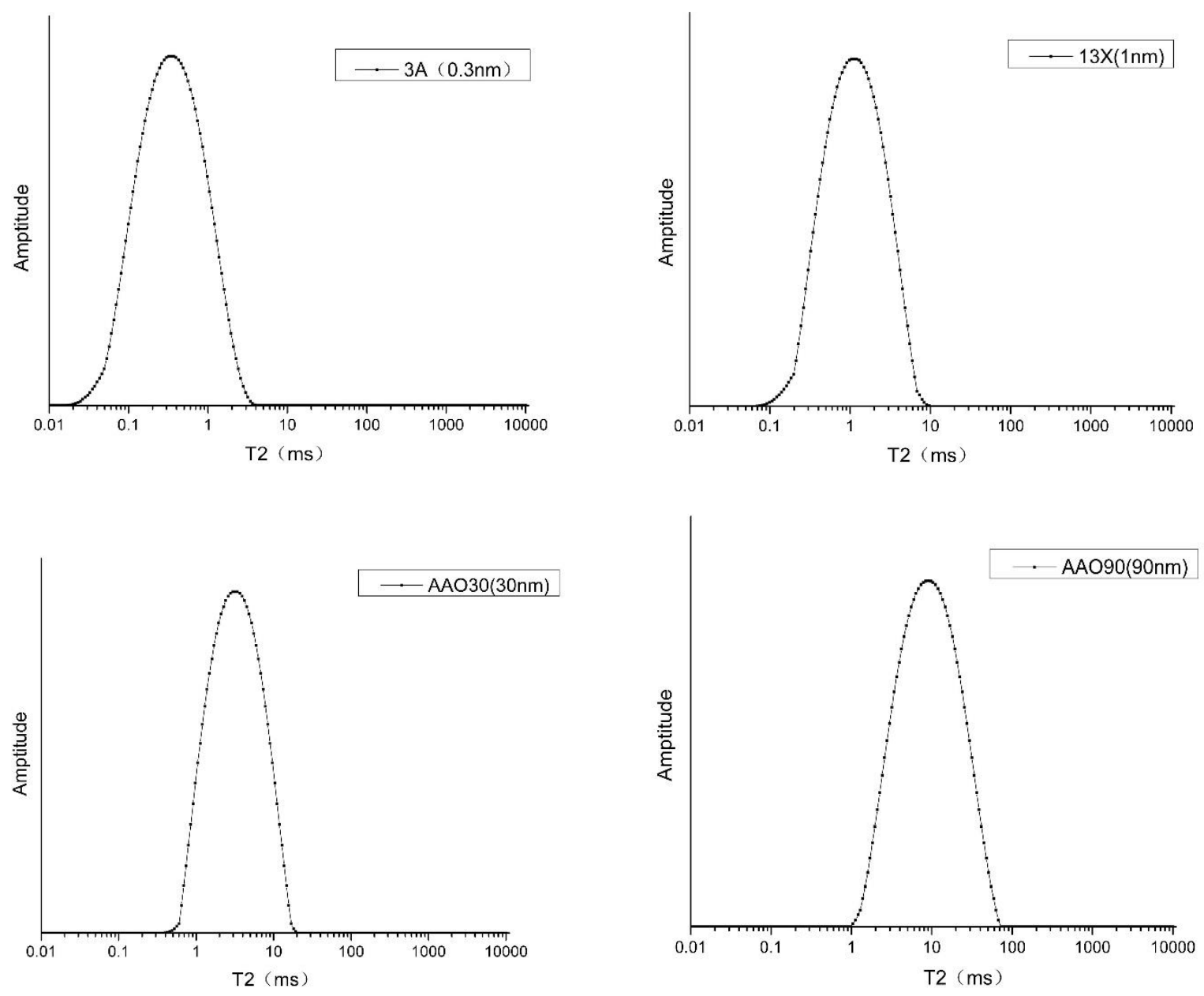

Fig. 1. The T2 spectrum after water absorption saturation of (a) $3 A(0.3 \mathrm{~nm})$, (b) $13 x(1 \mathrm{~nm})$, (c) AAO30 (30 nm), and (d) AAO90 $(90 \mathrm{~nm})$ molecular sieves. The spectra were inverted with CONTIN software and fitted with ORIGIN software.

The obtained surface relaxations were $3 \mathrm{~A} \rho=0.218 \mathrm{~nm} / \mathrm{ms}, 13 \mathrm{X} \rho=0.2159 \mathrm{~nm} / \mathrm{ms}$, AAO30 $\rho=2.471 \mathrm{~nm} / \mathrm{ms}$, and AAO $90 \rho=2.497 \mathrm{~nm} / \mathrm{ms}$. For $13 \mathrm{X}$ zeolite $/ 3 \mathrm{~A}$ zeolite, the relaxation time ratio of T2 peak apex was 3.379, and for AAO90 / AAO30, the relaxation time ratio of $\mathrm{T} 2$ peak apex was 3.031. The actual pore ratios of the samples were 3.334 for 13X / 3A and 3.000 for AAO90 / AAO30. Comparing the pore diameter calculated from the T2 spectrum with the actual pore diameter of the sample, it can be found that the results of the two were within an acceptable range, which indicates that the pore size result reflected by the relaxation time of the $\mathrm{T} 2$ spectrum can be used as a pore size detection method.

Table 2 shows that the surface relaxation rates of the same type of zeolite materials were basically the same, but the surface relaxation rates of different materials are very different due to the different adsorption forces of the inner walls of the pores on the liquid. Surface relaxation between identical materials is affected by pore size; smaller pores result 
in larger surface relaxation rates. This shows that the T2 spectrum can reflect the pore size of the material and is related to the relaxation rate of the material.

Table 1. Peak Top Values of Spectrum

\begin{tabular}{|c|c|c|c|c|}
\hline $\begin{array}{c}\text { Number of } \\
\text { measurements }\end{array}$ & $3 \mathrm{~A}(0.3 \mathrm{~nm})$ & $13 \times(1 \mathrm{~nm})$ & AAO30 $(30 \mathrm{~nm})$ & AAO90 $(90 \mathrm{~nm})$ \\
\hline 1 & 0.321 & 1.177 & 2.979 & 9.221 \\
\hline 2 & 0.372 & 1.056 & 3.169 & 9.207 \\
\hline 3 & 0.403 & 1.198 & 2.979 & 9.023 \\
\hline 4 & 0.321 & 1.193 & 2.813 & 9.165 \\
\hline 5 & 0.292 & 1.127 & 3.185 & 8.996 \\
\hline 6 & 0.313 & 1.212 & 2.851 & 9.207 \\
\hline 7 & 0.322 & 1.131 & 3.147 & 9.09 \\
\hline 8 & 0.398 & 1.173 & 2.911 & 8.928 \\
\hline
\end{tabular}

Table 2. Average Value of T2 Apex, Surface Relaxivity, Proportion of T2 Peak Apex, and Proportion of Pore Size

\begin{tabular}{|c|c|c|c|c|}
\hline Sample Name & $\begin{array}{c}\text { The Average } \\
\text { Value of } T 2 \text { Peak } \\
\text { Apex }\end{array}$ & $\begin{array}{l}\text { Surface } \\
\text { Relaxivity } \\
\text { (nm/ms) }\end{array}$ & $\begin{array}{c}\text { Proportion of } T 2 \\
\text { Peak Apex }\end{array}$ & $\begin{array}{c}\text { The Aperture } \\
\text { Ratio }\end{array}$ \\
\hline $3 \mathrm{~A}(0.3 \mathrm{~nm})$ & 0.342 & 0.218 & \multirow{2}{*}{3.379} & \multirow{2}{*}{3.334} \\
\hline $13 X(1 \mathrm{~nm})$ & 1.158 & 0.2159 & & \\
\hline AAO30 $(30 \mathrm{~nm})$ & 3.004 & 2.497 & \multirow{2}{*}{3.031} & \multirow{2}{*}{3.000} \\
\hline AAO90 $(90 \mathrm{~nm})$ & 9.105 & 2.471 & & \\
\hline
\end{tabular}

\section{CONCLUSIONS}

1. The surface relaxivity of the material is converted from the single pore size material. The surface relaxivity of zeolite and that of AAO porous membrane are quite different, but the surface relaxivity of the same material is the same. The surface relaxivity of the substance is related to its property and the size of pore. When the pore of the substance is a micropore, the smaller the pore is, the stronger the surface bondage received by the content is, and the faster the surface relaxivity occurs.

2. The zeolite molecular sieve material itself has strong water absorption characteristics, and the zeolite molecular sieve sample has a smaller pore size, which indicates that the pore inner surface of the zeolite molecular sieve sample has a stronger adsorption force with water, and the surface relaxation rate is related to the pore size. There is a strong relationship between adsorption strength, which is worthy of further discussion.

3. The pore size of porous materials can be calculated by the relationship between the $T 2$ spectrum and surface relaxivity. The proportion of the peak apex of the $T 2$ spectrum tends to be consistent with the proportion of pore size, which contributes greatly to the characterization of pore information of porous materials using TD NMR technology. The method of calculating the surface relaxation rate by $T 2$ spectrum calculation can be studied in depth, and thus becomes a method of pore characterization. 


\section{ACKNOWLEDGMENTS}

This research is supported by the National Natural Science Foundation of China (31860185/C160301).

\section{REFERENCES CITED}

Ajayan, V., Toshiyuki, M., and Katsuhiko, A. (2006). "New families of mesoporous materials," Science and Technology of Advanced Materials 7(8), 753-771. DOI: 10.1016/j.stam.2006.10.007

Akoka, S., Barantin, L., and Trierweiler, M. (1999). "Concentration measurement by proton NMR using the ERETIC method," Analytical Biochemistry 71(13), 25542557. DOI: $10.1021 /$ ac981422i

Bin, F. (2014). Dynamic Mechanical Properties and Microscopic Mechanism of Rock under Freezing and Thawing Cycles, Central South University, Changsha, China.

Bolton, A. J., Dinwoodie, J. M., and Davies, D. A. (1988). "The validity of the use of SEM/EDXA as a tool for the detection of UF resin penetration into wood cell walls of particleboard," Wood Science Technology 22(4), 345-356. DOI: 10.1007/BF00353324

Bucur, V. (2003). Nondestructive Characterization and Imaging of Wood, SpringerVerlag, Berlin.

Cavallaro, G., Donato, D. I., Lazzara, G., and Milioto, S. (2013). "Determining the selective impregnation of waterlogged archaeological woods with poly(ethylene) glycols mixtures by differential scanning calorimetry," Journal of Thermal Analysis Calorimetry 111(2), 1449-1455. DOI: 10.1007/s10973-012-2528-7

Deng, K. (2010). Nuclear Magnetic Resonance Logging Theory and Application, China University of Petroleum Press, Dongying, China.

Everett, D. H. (1972). "Manual of symbols and terminology for physicochemical quantities and units, Appendix II: Definitions, terminology and symbols in colloid and surface chemistry," Pure and Applied Chemistry 31(4), 577-638. DOI: 10.1351/pac197231040577

Furuno, T., Imamura, Y., and Kajita, H. (2004). "The modification of wood by treatment with low molecular weight phenol-formaldehyde resin: A properties enhancement with neutralized phenolic-resin and resin penetration into wood cell walls," Wood Science Technology 37(5), 349-361. DOI: 10.1007/s00226-003-0176-6

Gierling, N., Hansmann, C., Röder, T., Sixta, H., Gindl, W., and Wimmer, R. (2005). "Comparison of UV and confocal Raman microscopy to measure the melamineformaldehyde resin content within cell walls of impregnated spruce wood," Holzforschung 59(2), 210-213. DOI: 10.1515/HF.2005.033

Gindl, W., Zargar-Yaghubi, F., and Wimmer, R. (2003). "Impregnation of softwood cell walls with melamine- formaldehyde resin," Bioresources Technology 87(3), 325-330. DOI: 10.1016/S0960-8524(02)00233-X

Halperin, W. P., Jehng, J. Y., and Song, Y. Q. (1994). “Application of spin-spin relaxation to measurement of surface area and pore size distributions in a hydrating cement paste," Magnetic Resonance Imaging 12(2), 169. DOI: 10.1016/0730725X(94)91509-1

He, Y., Mao, Z., Xiao, L., and Ren, X. J. (2013). “An improved method for evaluating 
rock pore size distribution by NMR T2 distribution," Chinese Journal of Geophysics (02). DOI: $10.1002 / \mathrm{cjg} 2.668$

Hill, C. A. S. (2006). Wood Modification: Chemical, Thermal and Other Processes, John Wiley \& Sons, Hoboken, NJ, USA.

Jackson, C., and Makenna, G. B. (1990). "The melting behavior of organic materials confined in porous solids," The Journal of Chemical Physics 1990,93(12):9002. DOI: $10.1063 / 1.459240$

Jeremic, D., and Cooper, P. (2009). "PEG quantification and examination of molecular weight distribution in wood cell walls," Wood Science Technology 43(3-4), 317-329. DOI: $10.1007 / \mathrm{s} 00226-008-0233-2$

Jia, X. T., He, W., and Zhang, X. D. (2007). " $\mathrm{N}_{2}$ adsorption curve of meso-porous $\mathrm{SiO}_{2}$ : A neglected characterization technique," Materials Letters 61, 4377. DOI: 10.1016/j.matlet.2007.02.006

Jiang, Z., Deng, Z., and Zhang, N. (2012a). "The pore size method is used to characterize the pore structure of cement-based materials," Journal of the Chinese Academy of Ceramics 40(8), 1081-1087.

Jiang, Z., Deng, Z., Yuan, Z., and Li, W. (2013). "Thermoporometry to characterize the structure of concrete pores at different ages," Journal of Building Materials 16(6), 1044-1048. DOI:10.3969/j.issn.1007-9629.2013.06.021

Jiang, Z., Zhang, N., and Yang, Z. (2012b). "Thermodynamic calculation model for characterizing pore structure of cement-based materials by thermowell method," Journal of the Chinese Academy of Ceramics 40(2), 194-199.

Kubik, J. (1992). "Pore structure in dynamic behaviour of saturated materials," Transport in Porous Media 9(1-2), 15-24. DOI: 10.1007/BF01039622

Levitz, P.T. (2007). "Toolbox for 3D imaging and modeling of porous media: Relationship with transport properties," Cement and Concrete Research 37(3), 351359. DOI: 10.1016/j.cemconres.2006.08.004

Li, H., Zhu, J., and Guo, H. (2008). "Study on the method of NMR radius T2 spectrum conversion pore radius distribution," Chinese Journal of Spectroscopy 25(2), 273280.

Li, X., Li, Y., Chen, C., Zhao, D., Wang, X., Zhao, L., Shi, H., Ma, G., and Su, Z. (2015). "Pore size analysis from low field NMR spin-spin relaxation measurements of porous microspheres," Journal of Porous Materials 22(1), 11-20. DOI: 10.1007/s10934-0149864-x

Mitchell, J., Webber, B., and Strange, J. H. (2008). "Nuclear magnetic resonance cryoporometry," Physics Reports 461(1), 1-36. DOI: 10.1016/j.physrep.2008.02.001

Moura, M. J., Ferreira, P., and Figueiredo, M. M. (2005). "Mercury intrusion porosimetry in pulp and paper technology," Powder Technology 160(2), 61-66. DOI: 10.1016/j.powtec.2005.08.033

Nakamura, K., Hatakeyama, T., and Hatakeyama, H. (1981). Studies on Bound Water of Cellulose by Differential Scanning Calorimetry, University of the Philippines at Los Banos.

Notley, S. M., and Wagberg, L. (2005). "Morphology of modified regenerated model cellulose II surfaces studied by atomic force microscopy: Effect of carboxymethylation and heat treatment," Biomacromolecules 6(3), 1586-1591. DOI: $10.1021 / \mathrm{bm} 050005 \mathrm{u}$

Papadopoulos, A. N., Hill, C. A. S., and Gkaraveli, A. (2003). "Determination of surface area and pore volume of holocellulose and chemically modified wood flour using the 
nitrogen adsorption technique," Holz als Roh- und Werkstoff 61(6), 453-456. DOI: 10.1007/s00107-003-0430-5

Park, S., Venditti, R. A., and Jameel, H. (2006). "Changes in pore size distribution during the drying of cellulose fibers as measured by differential scanning calorimetry," Carbohydrate Polymers 66(1), 97-103. DOI: 10.1016/j.carbpol.2006.02.026

Pauli, G.F., Jaki, B.U., and Lankin, D.C. (2005). "Quantitative 1H NMR: Development and potential of a method for natural products analysis," Journal of Natural Products 68(1), 133-149.. DOI: 10.1021/np0497301

Plötze, M., and Niemz, P. (2011). "Porosity and pore size distribution of different wood types as determined by mercury intrusion porosimetry," European Journal of Wood and Wood Products 69(4), 649-657. DOI: 10.1007/s00107-010-0504-0

Provencher, S. W. (1982). "CONTIN: A general purpose constrained regularization program for inverting noisy linear algebraic and integral equations," Computer Physics Communications 27(3), 229-242. DOI: 10.1016/0010-4655(82)90174-6

Roels, S., Elsen, J., Carmeliet, J., and Hens, H. (2001). "Characterization of pore structure by combining mercury porosimetry and micrography," Materials and Structures 34(3), 76-82. DOI: 10.1007/BF02481555

Song, Y. Q. (2007). "Novel NMR techniques for porous media research," Cement and Concrete Research 37, 325. DOI: 10.1016/j.cemconres.2006.02.013

Stein, A., Wang, Z. Y., and Fierke, M. A. (2008). "Functionalization of porous carbon materials with designed pore architecture," Advanced Materials 20, 1. DOI: 10.1002/adma.200801492

Telkki, V. V. (2012). "Wood characterization by NMR and MRI of fluids," eMagRes (1), 215-222. DOI: 10.1002/9780470034590.emrstm1298

Telkki, V. V., Yliniemi, M., and Jokisaari, J. (2013). "Moisture in softwoods: Fiber saturation point, hydroxyl site content, and the amount of micropores as determined from NMR relaxation time distributions," Holzforschung 67(3), 291-300. DOI: 10.1515/hf-2012-0057

Teng, Y., Lian, S., and Song, T. (2014). "Change of relaxation time and pore structure during in situ low temperature drying of Shengli lignite based on -1H-NMR [J]," Chinese Journal of Coal (12): 2525-2530.

Topgaard, D., and Soderman, O. (2002). "Self-diffusion of nonfreezing water in porous carbohydrate polymer systems studied with nuclear magnetic resonance," Biophysical Journal 83(6), 3596-3606. DOI: 10.1016/S0006-3495(02)75360-5

Toumelin, E., Torres-Verdín, C., Boqin, S., and Dunn, K. J. (2007). "Random-walk technique for simulating NMR measurements and 2D NMR maps of porous media with relaxing and permeable boundaries," Journal of Magnetic Resonance 188(1), 8396. DOI: 10.1016/j.jmr.2007.05.024

Walker, J. C. F. (2006). "Chapter 3: Water in wood," in: Primary Wood Processing Principles and Practice ( $2^{\text {nd }}$ ed.), Springer, Berlin, pp. 69-74.

Wallström, L., and Lindberg, K. A. H. (1999). "Measurement of cell wall penetration in wood of water-based chemicals using SEM/EDS and STEM/EDS technique," Wood Science Technology 33(2), 111-122. DOI: 10.1007/s002260050103

Wang, X., and Liu, J. (2006). "The application of atomic force microscopy in wood science research," World Forestry Research 19(1), 38-41. DOI: 10.3969/j.issn.10014241.2006.01.009

Wang, Z., and Wang, W. (2013). "The principle and application of nuclear magnetic resonance freezing method are briefly described," Materials Review 27(1), 129-133. 
DOI: 10.3969/j.issn.1005-023X.2013.01.026

Wang, Z., Huang, C., and Wang, W. (2012). "Measurement of pore size distribution of cement-based materials by nuclear magnetic resonance freezing method," Journal of Building Materials 16(1), 6-10. DOI: 10.3969/j.issn.1005-023X.2013.01.026

Westermarck, S. (2000). Use of Mercury Porosimetry and Nitrogen Adsorption in Characterization of the Pore Structure of Mannitol and Microcrystalline Cellulose Powders, Granules, and Tablets, University of Helsinki, Helsinki, Finland.

Xiao, L. (1998). Magnetic Resonance Imaging Logging and Rock Nuclear Magnetic Resonance and Its Application, Science Press, Beijing, China..

Xu, F., Shi, Y.C., and Wang, D. (2013). "X-ray scattering studies of lignocellulosic biomass: a review," Carbohydrate Polymers 94(2), 904-917.

DOI: $10.1016 /$ j.carbpol.2013.02.008

Yamamoto, T., Endo, A., Inagi, Y., Ohmori, T., and Nakaiwa, M. (2005). "Evaluation of thermoporometry for characterization of mesoporous materials," Journal of Colloid and Interface Science 284(2), 614-620. DOI: 10.1016/j.jcis.2004.10.025

Zhao, L. (2010). Optical Constant and Wetting Characteristics of Porous Anodic Alumina Films, Northwest Normal University, Lanzhou, China.

Zhao, L. (2010). Research and Application of Nuclear Magnetic Resonance in the Determination of Reservoir Physical Properties, China University of Petroleum, Qingdao, China.

Zhao, Y., Chen, S., and Guo, Z. (2006). "Application of nuclear magnetic resonance method in pore structure of tight sandstone reservoir: A case study of the Member 3 of the Upper Paleozoic Shihezi formation in the Daniudi gas field," Ordos 01, 019112. DOI: $10.3969 /$ j.issn. 1000-7849.2006.01.020

Zhu, J., Xi, Z., Tang, H., and Ping, T. (2006). "Porous structure characterization and fractal theory research profile," Rare Metal Materials and Engineering 35(S2), 452456. DOI: 10.3321/j.issn:1002-185X.2006.z1.109

Article submitted: August 30, 2019; Peer review completed: October 19, 2019; Revised version received: December 9, 2019; Accepted: December 10, 2019; Published: January $8,2020$.

DOI: 10.15376/biores.15.1.1407-1418 